\title{
Complete asymptotically safe embedding of the standard model
}

\author{
Steven Abel, ${ }^{1, *}$ Esben Mølgaard, ${ }^{2, \dagger}$ and Francesco Sannino, $2,3, \$$ \\ ${ }^{1}$ IPPP, Durham University, South Road, Durham, DH1 3LE, United Kingdom \\ ${ }^{2} \mathrm{CP} P^{3}$-Origins and the Danish Institute for Advanced Study, University of Southern Denmark, \\ Campusvej 55, DK-5230 Odense, Denmark \\ ${ }^{3}$ SLAC, National Accelerator Laboratory, Stanford University, Stanford, California 94025, USA
}

(Received 2 January 2019; published 19 February 2019)

\begin{abstract}
We present and discuss the "Tetrad Model", a large color/flavor embedding of the Standard model which has an interacting ultraviolet fixed point. It is shown that its extended-Pati-Salam symmetry is broken radiatively via the Coleman-Weinberg mechanism, while the remaining electroweak symmetry is broken when mass-squared terms run negative. In the IR the theory yields just the Standard Model, augmented by the fact that the Higgs fields carry the same generation indices as the matter fields. It is also shown that the Higgs mass-squareds develop a hierarchical structure in the IR, from a UV theory that is asymptotically flavor symmetric, opening up an interesting direction for explaining the emergence of the observed flavor structure.
\end{abstract}

DOI: 10.1103/PhysRevD.99.035030

\section{INTRODUCTION AND OVERVIEW OF EMBEDDING}

A recent series of papers [1,2] suggested a framework for embedding the Standard Model (SM) in an asymptotically safe ultraviolet (UV) completion [3]. (For some related discussions of asymptotic safety applications see [4-22]. For recent reviews see [22-24].) The framework is partially perturbative based on the weak ultraviolet (UV) fixed points of $[12,16,17]$ (hereafter LS). These are the UV counterparts of the better known Caswell-Banks-Zaks infrared (IR) fixed points, in a large color and flavor Veneziano limit. By suitable adjustment of the numbers of colors and flavors, a UV fixed point can be achieved that is arbitrarily weakly coupled. Coupled with the "large flavor" fixed points of [25-30] operating for the electroweak gauge couplings, one finds an asymptotically safe extension of the Pati-Salam (PS) theory, that has a UV fixed point with a gauge group $S U\left(N_{C}\right) \times S U(2)_{L} \times S U(2)_{R}$ and a natural breaking down to the SM gauge group in the IR driven partially by radiative symmetry breaking. The main observation of [2] was that the two kinds of fixed points (Veneziano and large $N_{f}$ ) do not interfere with each other.

\footnotetext{
s.a.abel@durham.ac.uk †molgaard@cp3-origins.net

*sannino@cp3-origins.net
}

Published by the American Physical Society under the terms of the Creative Commons Attribution 4.0 International license. Further distribution of this work must maintain attribution to the author(s) and the published article's title, journal citation, and DOI. Funded by SCOAP .
Despite this attractive framework for embedding the Standard Model, the theories presented in [1,2] did not provide a mechanism for fully removing the extraneous degrees of freedom (d.o.f.) in the IR to leave purely the SM. In particular in this simplest realization, there remain in the low energy theory a large multiplicity of electroweak $S U(2)$ doublets that are unmatched and hence massless.

In this paper we provide a complete phenomenological framework, by an enhancement that yields a theory flowing from an asymptotically safe fixed point in the UV to precisely the SM, augmented only by additional Higgses. In particular there are no other light superfluous states remaining in the theory. The additional Higgses are furnished with the same generation numbers as the matter fields, so their vacuum expectation values (VEVs) may therefore ultimately be able to explain flavor hierarchies (although we do not attempt this in the present paper).

Moreover symmetry breaking can be entirely radiative. It can happen in two ways (or by a combination of them). One possibility is the traditional radiative symmetry breaking mechanism of Coleman and Weinberg [31-33]. This can be shown to occur analytically driven by a single quartic coupling running negative and generating a minimum according to the pattern discussed in [32]. This can be responsible for the bulk of the breaking of the extended PS gauge group. At the same time the PS breaking generates a positive masssquared for the Higgs at the high scale due to a portallike coupling between the electroweak Higgs and the PS Higgs. This can run negative in the IR due to large Yukawa couplings from its initially positive boundary value at the PS-scale. The alternative possibility is that radiative symmetry breaking is instead dominated by the dimensionful couplings 
(i.e., mass-squared terms) and the PS breaking minimum is generated radiatively when they run negative due to the large Yukawa couplings that have to be present in the LS gaugeYukawa theories. This was the mechanism discussed in [1], which is essentially the asymptotically safe version of the radiative symmetry breaking in the supersymmetric Standard Model [34]. Thus one appears to have the freedom to turn on as much or as little of the classically dimensionful operators as desired in the symmetry breaking.

In order to present our model we will also advocate in this paper the use of "quiver" diagrams. Such diagrams can greatly alleviate the generic problem that the UV of asymptotically safe models is complicated because they necessarily have to include extra d.o.f., and as a consequence the structure is often hard to appreciate (or present), even though it may in reality be relatively simple. Although (depending on the model in question) it may only be possible to represent part of the gauge groups in quiver diagrams (if some of the states do not easily fall into bifundamentals), their use can greatly ease the construction of phenomenologies within an asymptotically safe framework.

\section{THE “TETRAD” MODEL (TM)}

\section{A. Structure in the UV}

We begin by recapping the LS fixed point of [16], whose field content is shown in Table I, where midalphabet latin indices $i, j, k \ldots$ are used to label flavor, while earlyalphabet latin indices $a, b, c \ldots$ label color. The particle content is represented as in a conventional quiver diagram, in Fig. 1. As usual, the circular nodes represent the $S U\left(N_{C}\right)$ gauge factor, which is crucial in establishing the LS fixed point. The square nodes represent the flavor groups, $S U\left(N_{F}\right)_{L} \otimes S U\left(N_{F}\right)_{R}$, which will become partially gauged in order to accommodate the electroweak gauge factors of the SM. In [1,2] the LS model was augmented by colored scalars in order to break the gauge group down to the SM. However as mentioned in the Introduction, there remain in such models light doublets which are charged under the electroweak SM gauge groups.

Let us now proceed directly to the phenomenologically viable augmented model that we will propose in this paper. As we shall see the model leaves no light states, other than those appearing directly in the SM, beyond an enhanced Higgs sector (with the Higgs fields carrying the same

TABLE I. Fields in the arbitrarily weakly coupled asymptotic safe fixed point of [16].

\begin{tabular}{ccccc}
\hline \hline & $S U\left(N_{C}\right)$ & $S U\left(N_{F}\right)_{L}$ & $S U\left(N_{F}\right)_{R}$ & Spin \\
\hline$Q_{a i}$ & $\square$ & $\square$ & 1 & $1 / 2$ \\
$\tilde{Q}^{i a}$ & $\tilde{\square}$ & 1 & $\tilde{\square}$ & $1 / 2$ \\
$H_{j}^{i}$ & 1 & $\tilde{\square}$ & $\square$ & 0 \\
\hline \hline
\end{tabular}

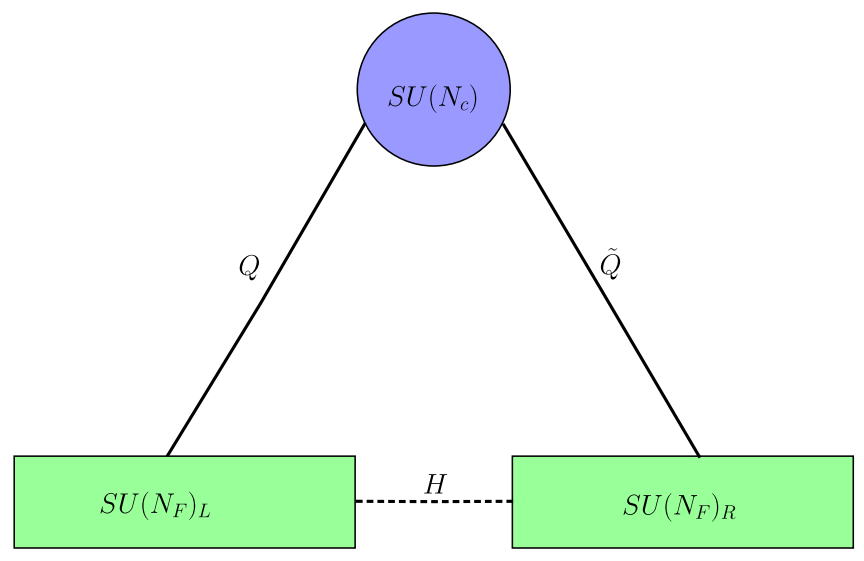

FIG. 1. Quiver diagram of the fixed point theory of [16]. Solid lines represent fermions; dashed lines represent bosons.

generation indices as the matter fields). In this section we will lay out the spectrum and pattern of VEVs that need to be achieved in order to realise the Standard Model in the IR, and then in the following section we consider the dynamics that achieves them. The augmented model is shown in Table II and its corresponding quiver diagram in Fig. 2. It contains four elements; hence we refer to it as the Tetrad Model (TM $\left.{ }^{\mathrm{TM}}\right)$. As in [2] it is an extension of the PS model to a larger unified group. Note that the PS gauge unification to $S U(2)_{R}$ is adopted to take advantage of the $S U(2)$ largeflavor fixed points, introduced in $[25,26]$. We will use a Weyl notation and display the left and right fermions explicitly. We use the following nomenclature for the spectrum: Fermions will be denoted with $Q$ and $q$ 's, while scalars will be denoted with $\tilde{S}$ and $H$ 's. ${ }^{1}$ The flavor indices $i=$ $1 \ldots N_{F}$ have three generations of components gauged under electroweak $S U(2)_{L}$ and $S U(2)_{R}$. However we have to gauge the right-handed component of the electroweak gauge group in the correct way to yield the SM spectrum. Indeed the "squarks" $\tilde{S}$ have their own $S U\left(N_{S}\right)$ flavor symmetry, and the first two flavors also have to be charged under $S U(2)_{R}$ in order to give the correct PS breaking. The simplest solution is then to identify $S U(2)_{R}=\left[S U(2)_{r} \otimes\right.$ $\left.S U(2)_{S}\right]_{\text {diag }}$. This leads to hypercharge $Y=\left(2 T_{R}^{(3)}+B-L\right)$ and charge $Q_{\text {e.m. }}=\frac{1}{2}\left(2 T_{R}^{(3)}+2 T_{L}^{(3)}+B-L\right)$, where $T_{L / R}^{(3)}=$ $\operatorname{diag}\left(\frac{1}{2},-\frac{1}{2}\right)$ and $B-L$ is the $\operatorname{diag}\left(\frac{1}{3}, \frac{1}{3}, \frac{1}{3},-1,0,0 \ldots, 0\right)$ generator of $S U\left(N_{C}\right)$. As we shall see, for the LS gaugeYukawa fixed point to be weakly coupled we require $N_{F} \approx \frac{21}{4} N_{C}$.

The necessity of the additional fermionic fields $q, \tilde{q}$ can be deduced from the requirement that they are able to remove the unwanted light fermionic d.o.f. while maintaining the chiral symmetry. The allowed

\footnotetext{
${ }^{1}$ The $\tilde{S}$ scalars were referred to as $\tilde{Q}$ in [2], but in the present context this would cause confusion.
} 
TABLE II. Fields in the asymptotically safe "Tetrad" Model, where $N_{S}=N_{C}-2$ and $N_{F} \approx \frac{21}{4} N_{C}$. The top $2 n_{g}=6$ components of flavor $S U\left(N_{F}\right)$ correspond to $S U(2)$ multiplets, where $n_{g}$ is the generation number. The gauging for the usual Pati-Salam $S U(2)_{R}$ group is identified as $S U(2)_{R}=\left[S U(2)_{r} \otimes S U(2)_{S}\right]_{\text {diag }}$.

\begin{tabular}{lccccc}
\hline \hline & $S U\left(N_{C}\right)$ & $S U\left(N_{F}\right)_{L} \supset S U(2)_{L} \otimes S U\left(n_{g}\right)_{L}$ & $S U\left(N_{F}\right)_{R} \supset S U(2)_{r} \otimes S U\left(n_{g}\right)_{r}$ & $S U\left(N_{S}\right)=S U\left(N_{C}-4\right)_{S} \oplus S U(2)_{S}$ & Spin \\
\hline$Q_{a i}$ & $\square$ & $\square \supset(\square, \square)$ & 1 & 1 & $1 / 2$ \\
$\tilde{Q}^{i a}$ & $\tilde{\square}$ & 1 & $\tilde{\square}(\tilde{\square}, \tilde{\square})$ & 1 & $1 / 2$ \\
$H_{j}^{i}$ & 1 & $\tilde{\square}(\tilde{\square}, \tilde{\square})$ & $\square \supset(\square, \square)$ & 1 & 0 \\
$\tilde{S}_{a, \ell=1 . . N_{S}}$ & $\tilde{\square}$ & 1 & 1 & $\tilde{\square}=\tilde{\square}_{N_{C}-4} \oplus \tilde{\square}_{2}$ & 0 \\
$\tilde{q}_{\ell}^{i}$ & 1 & $\tilde{\square}(\tilde{\square}, \tilde{\square})$ & 1 & $\square=\square_{N_{C}-4} \oplus \square_{2}$ & $1 / 2$ \\
$q_{j}^{\ell}$ & 1 & 1 & $\square \supset(\square, \square)$ & $\tilde{\square}=\tilde{\square}_{N_{C}-4} \oplus \tilde{\square}_{2}$ & $1 / 2$ \\
\hline \hline
\end{tabular}

couplings one can consider for the generation of the UVfixed point are

$$
\begin{aligned}
\mathcal{L}_{\mathrm{UVFP}} \supset & \mathcal{L}_{\mathrm{KE}}+\frac{y}{\sqrt{2}} \operatorname{Tr}[(Q H) \cdot \tilde{Q}]+\frac{\tilde{y}}{\sqrt{2}} \operatorname{Tr}\left[q H^{\dagger} \tilde{q}\right] \\
& -\frac{\tilde{Y}}{\sqrt{2}} \operatorname{Tr}[(\tilde{S} \cdot Q) \tilde{q}]-\frac{Y}{\sqrt{2}} \operatorname{Tr}\left[\left(\tilde{Q} \cdot \tilde{S}^{\dagger}\right) q\right] \\
& -u_{1} \operatorname{Tr}\left[H^{\dagger} H\right]^{2}-u_{2} \operatorname{Tr}\left[H^{\dagger} H H^{\dagger} H\right] \\
& -v_{1} \operatorname{Tr}\left[H^{\dagger} H\right] \operatorname{Tr}\left[\tilde{S}^{\dagger} \cdot \tilde{S}\right]-w_{1} \operatorname{Tr}\left[\tilde{S}^{\dagger} \cdot \tilde{S}\right]^{2} \\
& -w_{2} \operatorname{Tr}\left[\tilde{S}^{\dagger} \cdot \tilde{S} \tilde{S}^{\dagger} \cdot \tilde{S}\right],
\end{aligned}
$$

where the trace is over the flavor indices and the dot refers to color contraction. As we shall see the $Y$ and $\tilde{Y}$ Yukawa couplings are responsible for giving masses to the unwanted d.o.f. in the IR once $\tilde{S}$ gets a VEV. They are written above somewhat schematically as clearly they

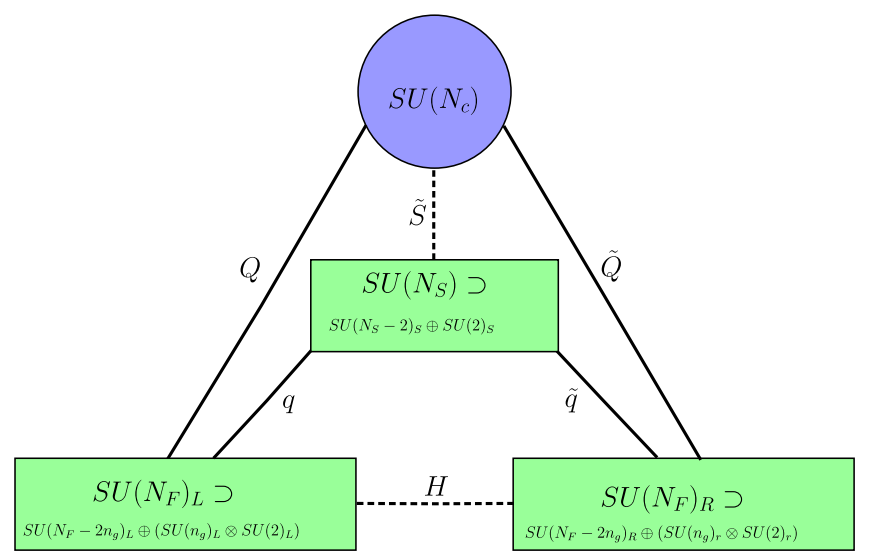

FIG. 2. The "Tetrad" quiver that gives the Standard Model in the IR. Note that is not possible to illustrate the gauging of the electroweak symmetries on such a diagram. On the right, the gauging is on the $S U(2)_{R}=\left[S U(2)_{r} \otimes S U(2)_{S}\right]_{\text {diag }}$ factor, with the top $2 n_{g}$ indices of $S U\left(N_{F}\right)_{L, R}$ flavor transforming as doublets under $S U\left(n_{g}\right) \otimes S U(2)_{L, R}$. cannot couple all the flavor components in the same way due to the $S U(2)_{R}$ gauge invariance. They will be treated explicitly below.

As in [2] we will not consider the flavor breaking coupling (schematically)

$$
\mathcal{L}_{S U\left(A_{F}\right)}=-v_{2} \operatorname{Tr}\left[H^{\dagger} H \tilde{S}^{\dagger} \cdot \tilde{S}\right] .
$$

This coupling can be fixed to be precisely zero, where it will remain along the flow. (It can of course be forbidden on grounds of preservation of flavor symmetry which we will associate with the classically relevant operators only.) As we shall see the flavor conserving portal coupling $v_{1}$ can generate a mass-squared for the electroweak Higgses, and we keep it in the analysis.

We can in addition include the aforementioned dimensionful "soft-terms". Unlike the classically dimensionless couplings these will be allowed to explicitly violate the flavor symmetry. They can be written most generally in the form

$\mathcal{L}_{\text {Soft }}=-m_{h_{0}}^{2} \operatorname{Tr}\left[H^{\dagger} H\right]-\sum_{a=1}^{N_{F}^{2}-1} \Delta_{a}^{2} \operatorname{Tr}\left[H T^{a}\right] \operatorname{Tr}\left[H^{\dagger} T^{a}\right]$,

where $T^{a}$ are the generators of the $S U\left(N_{F}\right)_{\text {diag }}$ flavor group. Being classically relevant, the soft terms cannot disrupt the UV fixed point, but can serve to generate symmetry breaking themselves, and also remove any Goldstone modes associated with the spontaneously broken global flavor symmetries.

\section{B. Structure in the IR-emergence of the SM from the TM}

Next let us confirm that the SM emerges in the IR from the Tetrad Model. It is useful for this purpose to explicitly write the particle content in terms of SM quantum numbers in order to discuss the couplings and determine the required values for $N_{F}, N_{S}$ : the explicit representations are (c.f. the usual PS model in e.g., [35]) 


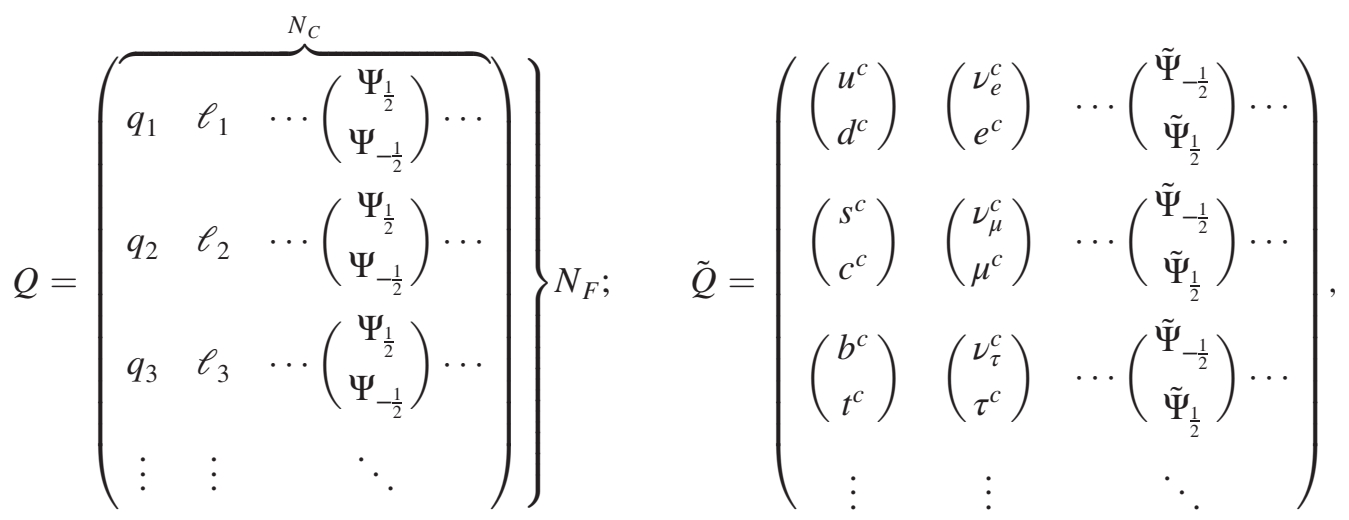

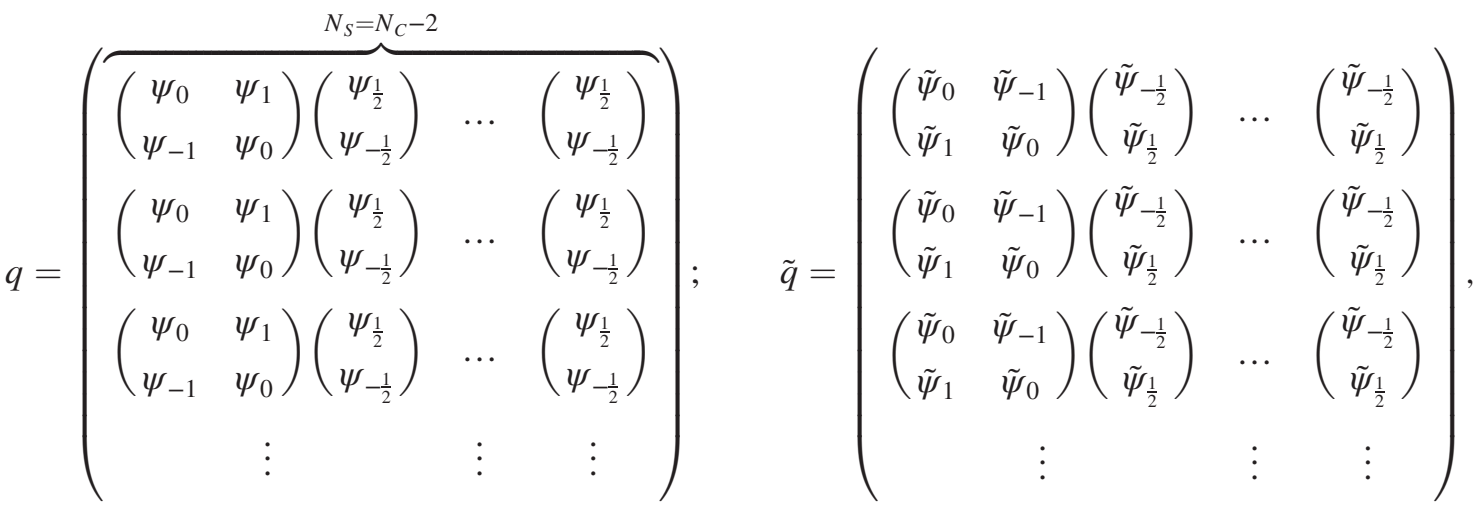

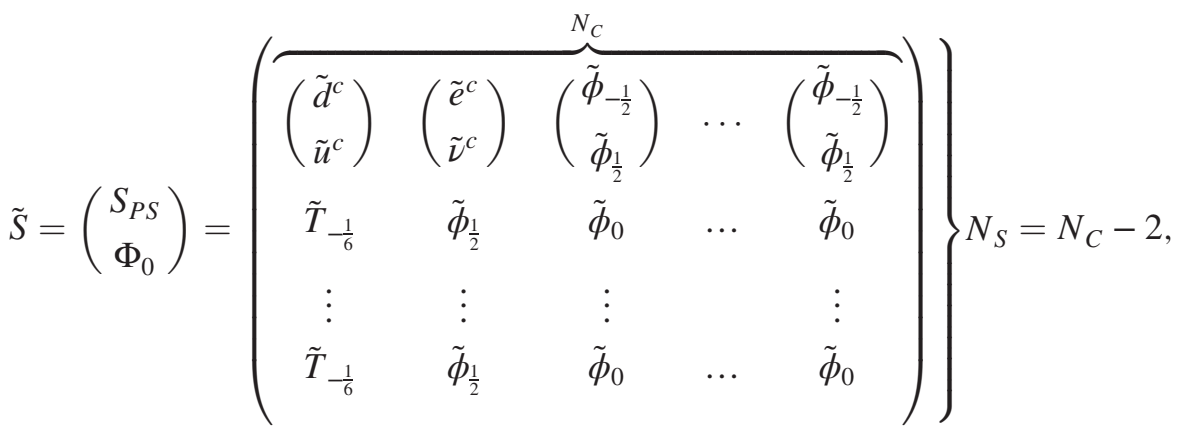

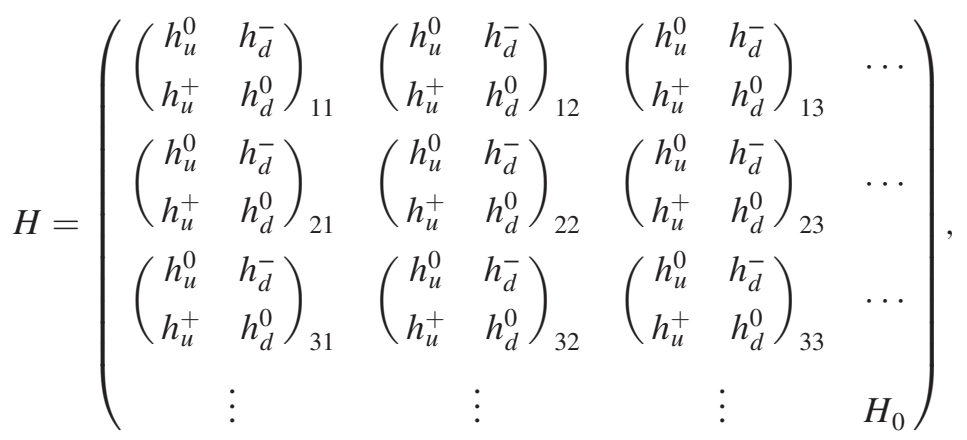

where $H_{0}$ is an $\left(N_{F}-6\right) \times\left(N_{F}-6\right)$ scalar which is uncharged under the SM gauge groups, and the sufficies denote $Q_{\text {e.m. }}$. The assignment of the remaining fields is obvious.

First note that the top $2 n_{g}$ (where $n_{g}=3$ is the number of generations, but it is often useful to leave it generic) entries of flavor are charged under the $S U(2)$ gauge groups. Therefore, given the couplings and matter content, there are $n_{g}$ generations of SM Higgs doublets in the top $2 n_{g} \times 2 n_{g}$ components of $H$. Assuming that $n_{g}=3$, this corresponds to 18 separate Higgs $S U(2)_{L}$ doublets. Clearly one ultimately requires these to be lifted in a hierarchical way so that there is one dominant lighter Higgs which gets a VEV, which will 
be a mixture of the 18 original ones. In contrast with [2] we will assume that the scalars $\tilde{S}$ are gauged only under color except for the first two flavors which are charged under the gauged $S U(2)_{R}$. (The latter choice is flexible.)

We repeat that we are assuming flavor degeneracy in all the couplings of (1). One could instead for example take the $y$ Yukawa couplings to break $S U\left(N_{F}\right)_{L} \times S U\left(N_{F}\right)_{R}$ symmetry, but this would require a reanalysis of the UV fixed point behavior of the theory so we instead adopt the philosophy of [2]. As there is a pair of Higgs multiplets for each generation, this is indeed an attractive possibility for introducing SMflavor structure. Moreover as shown in [2] and expanded upon below, the flavor universal part of such operators flows to relatively smaller absolute values, "exposing" flavor hierarchies during the flow, so that they become dominant in the IR.

There are two elements to the gauge symmetry breaking. First there are VEVs for $\tilde{S}$. We must choose $N_{S}=N_{C}-2$, so that they can be rearranged by suitable color and $S U\left(N_{S}\right)$ flavor rotations into the form

$\langle\tilde{S}\rangle=\tilde{V} \overbrace{\left(\begin{array}{cccccc}0 & 0 & 0 & 0 & \cdots & 0 \\ \vdots & \vdots & \vdots & 1 & & \vdots \\ \vdots & \vdots & \vdots & & \ddots & \vdots \\ 0 & 0 & 0 & 0 & \cdots & 1\end{array}\right)}^{N_{C}}\} N_{S}=N_{C}-2$

with the VEV $\tilde{\phi}_{0}$ in (6) being of the form $\left\langle\tilde{\phi}_{0}\right\rangle=\tilde{V} \mathbb{I}_{N_{C-4}}$, where $\tilde{V}$ is a constant. The $S U(2)_{R}$ orientation simply determines the direction corresponding to the massless right-handed "sneutrino", so one may always choose a basis in which the $\tilde{\nu}^{c}$ and $N_{C}-4$ of the $\tilde{\phi}_{0}$ 's on the diagonal get a VEV. (Obviously the case $N_{C}=4$ is the standard, nonasymptotically free, Pati-Salam model.)

At this stage the gauge symmetry is broken to the Standard Model as

$S U\left(N_{C}\right) \times S U(2)_{L} \times S U(2)_{R} \rightarrow S U(3)_{c} \times S U(2)_{L} \times U(1)_{Y}$.

Given that the gauge symmetry can be broken as required, one can focus on the excess states that need to be made massive in order to end up with the Standard Model in the IR. In particular there are of course (by design) very many $S U(2)_{L}$ and $S U(2)_{R}$ doublets that should be removed at low scales. The second component of symmetry breaking that accomplishes this is that the block $H_{0}$ of the Higgs multiplets also acquire VEV along the diagonal,

$$
\left\langle H_{0}\right\rangle=V_{0} \mathbb{I}_{N_{F}-6} .
$$

Thanks to the $y$ coupling, this gives the $N_{F}-6$ generations of complete nondoublet $S U\left(N_{C}\right)$ multiplets masses $\frac{y V_{0}}{\sqrt{2}}$, leaving untouched $n_{g}\left(N_{C}-4\right)$ of the $S U(2)_{L}$ doublets in the $Q_{L}$, and $S U(2)_{R}$ doublets in the $Q_{R}$. Indeed in these remaining $n_{g}$ generations of $S U\left(N_{C}\right)$-colored multiplets, only the first $S U(4)$ components are to be identified as matter fields, as in (4). The remaining states get masses $\frac{\tilde{Y} \tilde{V}}{\sqrt{2}}$ and $\frac{Y \tilde{V}}{\sqrt{2}}$ from the $\tilde{Y}$ and $Y$ couplings respectively to which we now return, writing them with explicit indices,

$$
\begin{aligned}
& \mathcal{L}_{\mathrm{UVFP}} \supset-\frac{\tilde{Y}}{\sqrt{2}} Q \tilde{S} \tilde{q}-\frac{Y}{\sqrt{2}} \tilde{S}^{\dagger} \tilde{Q} q, \\
& \supset-\frac{\tilde{Y}}{\sqrt{2}}\left(Q_{k}^{a \alpha} \tilde{S}_{a}^{j} \tilde{q}_{\alpha j}^{k}\right)-\frac{Y}{\sqrt{2}}\left(\tilde{Q}_{k}^{a \alpha} S_{a}^{* j} q_{\alpha j}^{k}\right),
\end{aligned}
$$

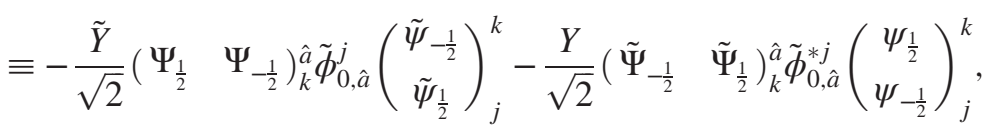

$$
\begin{aligned}
& =-\frac{\tilde{Y} \tilde{V}}{\sqrt{2}}\left(\begin{array}{ll}
\Psi_{\frac{1}{2}} & \Psi_{-\frac{1}{2}}
\end{array}\right)_{k}^{j}\left(\begin{array}{c}
\tilde{\psi}_{-\frac{1}{2}} \\
\tilde{\psi}_{\frac{1}{2}}
\end{array}\right)_{j}^{k}-\frac{Y \tilde{V}}{\sqrt{2}}\left(\begin{array}{ll}
\tilde{\Psi}_{-\frac{1}{2}} & \tilde{\Psi}_{\frac{1}{2}}
\end{array}\right)_{k}^{j}\left(\begin{array}{c}
\psi_{\frac{1}{2}} \\
\psi_{-\frac{1}{2}}
\end{array}\right)_{j}^{k},
\end{aligned}
$$

where $a=1 \ldots N_{C}$ are color indices, $\hat{a}=5 \ldots N_{C}$ are the $N_{C}-4$ color indices beyond the PS d.o.f., $j=1 \ldots N_{C}-4$ are the $N_{C}-4$ flavor indices of $\tilde{S}$ that are not charged under $S U(2)_{R}$, the indices $\alpha=1,2$ are the $S U(2)_{L / R}$ indices, and $k=1 \ldots n_{g}$ are generation indices. Note that as promised chiral symmetry dictates the choice $N_{S}=N_{C}-2$, because $N_{S}$ flavor is locked to $S U\left(N_{C}\right)$ color by the VEV of $\tilde{S}$. This leaves the first two columns of $q$ and $\tilde{q}$ which obtain their masses independently from only the $y, \tilde{y}$ terms in (1).

It is easy to check that with this choice of colors and flavors, and these VEVs, the remaining content in the IR is that of the SM with Higgses carrying $S U\left(n_{g}\right)$ generation indices for the left- and right-handed fields. 


\section{FLOW FROM THE UV FIXED POINTS AND SYMMETRY BREAKING}

\section{A. The Tetrad Model contains the Coleman Weinberg mechanism}

Next let us turn to the dynamics, first illustrating the appearance of traditional radiative symmetry breaking. As there are many couplings involved, it is useful to break down the evolution under renormalization group (RG) flow into self-contained units. Indeed the crucial aspect of the flow from the UV fixed point is that it is actually controlled by two fixed points of the gauge and Yukawa couplings, which form a closed system by themselves.

It will be convenient to define rescaled couplings as follows:

$$
\begin{array}{rlrl}
\alpha_{g} & =\frac{N_{C} g^{2}}{(4 \pi)^{2}} ; & \alpha_{y}=\frac{N_{C} y^{2}}{(4 \pi)^{2}} ; & \alpha_{\tilde{y}}=\frac{N_{C} \tilde{y}^{2}}{(4 \pi)^{2}} ; \\
\alpha_{Y}=\frac{N_{C} Y^{2}}{(4 \pi)^{2}} ; & \alpha_{\tilde{Y}}=\frac{N_{C} \tilde{Y}^{2}}{(4 \pi)^{2}} ; & \\
\alpha_{u_{1}}=\frac{N_{F}^{2} u_{1}}{(4 \pi)^{2}} ; & \alpha_{u_{2}}=\frac{N_{F} u_{2}}{(4 \pi)^{2}} ; & \alpha_{v_{1}}=\frac{N_{C}^{2} v_{1}}{(4 \pi)^{2}} ; \\
\alpha_{w_{1}}=\frac{N_{C}^{2} w_{1}}{(4 \pi)^{2}} ; & \alpha_{w_{2}}=\frac{N_{C} w_{2}}{(4 \pi)^{2}} . &
\end{array}
$$

To determine their fixed points, we require their RG equations to order $\alpha^{3} \equiv \epsilon \alpha^{2}$ in $\beta_{g}$ and order $\alpha^{2} \equiv \epsilon \alpha$ in $\beta_{y, Y, \tilde{Y}}$ : defining $\epsilon=-11 / 2+x_{F}+x_{q} / 4=x_{F}-21 / 4$ and $\Upsilon=\sqrt{\alpha_{y} \alpha_{\tilde{y}} \alpha_{Y} \alpha_{\tilde{Y}}}$, and taking $n_{g}=3$, the beta functions are found to be

$$
\begin{aligned}
\beta_{g}= & \alpha_{g}^{2}\left(\frac{4}{3} \epsilon+\left(\frac{26}{3} x_{F}-20\right) \alpha_{g}-x_{F}^{2} \alpha_{y}-x_{F} \alpha_{Y}-x_{F} \alpha_{\tilde{Y}}\right) \\
\beta_{y}= & 4 \Upsilon+\alpha_{y}\left(\left(1+x_{F}\right) \alpha_{y}+\alpha_{\tilde{y}}+\alpha_{\tilde{Y}}+\alpha_{Y}-6 \alpha_{g}\right) \\
\beta_{\tilde{y}}= & 4 \Upsilon+\alpha_{\tilde{y}}\left(\left(1+x_{F}\right) \alpha_{\tilde{y}}+\alpha_{y}+\alpha_{\tilde{Y}}+\alpha_{Y}\right) \\
\beta_{Y}= & 2 x_{F} \Upsilon+\alpha_{Y}\left(2\left(1+x_{F}\right) \alpha_{Y}\right. \\
& \left.+x_{F}\left(\frac{1}{2} \alpha_{y}+\frac{1}{2} \alpha_{\tilde{y}}+2 \alpha_{\tilde{Y}}\right)-3 \alpha_{g}\right) \\
\beta_{\tilde{Y}}= & 2 x_{F} \Upsilon+\alpha_{\tilde{Y}}\left(2\left(1+x_{F}\right) \alpha_{\tilde{Y}}\right. \\
& \left.+x_{F}\left(\frac{1}{2} \alpha_{y}+\frac{1}{2} \alpha_{\tilde{y}}+2 \alpha_{Y}\right)-3 \alpha_{g}\right) .
\end{aligned}
$$

Since it is positive, the equation for $\beta_{\tilde{y}}=0$ can only be consistently met with $\alpha_{\tilde{y}}=\Upsilon=0$. Moreover if any of the other couplings are nonzero, it flows to zero in the IR, so along the RG trajectory from any eligible fixed point it must remain zero. There are also by inspection no positive solutions with $\alpha_{y}=0$. In addition the last two equations
TABLE III. The collection of UV fixed points for the gauge and Yukawa couplings: schematically the flow is from $\mathrm{A} \rightarrow \mathrm{B} \rightarrow \mathrm{C}, \mathrm{D} \rightarrow \mathrm{E}$. Fixed points $\mathrm{C}, \mathrm{D}, \mathrm{E}$ are pseudofixed points in the sense that the quartic scalar coupings do not have a fixed point there. The only true nontrivial fixed point is the LS fixed point of B.

\begin{tabular}{lccccc}
\hline \hline Label & $\alpha_{g}^{*}$ & $\alpha_{\tilde{y}} / \alpha_{g}$ & $\alpha_{y} / \alpha_{g}$ & $\alpha_{Y} / \alpha_{g}$ & $\alpha_{\tilde{Y}} / \alpha_{g}$ \\
\hline $\mathrm{A}$ & 0 & 0 & 0 & 0 & 0 \\
$\mathrm{~B}$ & $\frac{25}{18} \epsilon$ & 0 & $\frac{6}{1+x_{F}} \rightarrow \frac{24}{25}$ & 0 & 0 \\
$\mathrm{C}$ & $\frac{302}{225} \epsilon$ & 0 & $\frac{6\left(3+4 x_{F}\right)}{4+7 x_{F}+4 x_{F}^{2}} \rightarrow \frac{144}{151} \frac{6}{4+7 x_{F}+4 x_{F}^{2}} \rightarrow \frac{6}{151}$ & 0 \\
$\mathrm{D}$ & $\frac{302}{225} \epsilon$ & 0 & $\frac{6\left(3+4 x_{F}\right)}{4+7 x_{F}+4 x_{F}^{2}} \rightarrow \frac{144}{151}$ & 0 & $\frac{6}{4+7 x_{F}+4 x_{F}^{2}} \rightarrow \frac{6}{151}$ \\
$\mathrm{E}$ & $\frac{277}{207} \epsilon$ & 0 & $\frac{6\left(1+4 x_{F}\right)}{2+5 x_{F}+4 x_{F}^{2}} \rightarrow \frac{264}{277} \frac{3}{2+5 x_{F}+4 x_{F}^{2}} \rightarrow \frac{6}{277} \frac{3}{2+5 x_{F}+4 x_{F}^{2}} \rightarrow \frac{6}{277}$ \\
\hline \hline
\end{tabular}

allow a fixed point if $\alpha_{Y}=\alpha_{\tilde{Y}}$ or one or both couplings vanish. Hence one finds the possible flows shown in Table III (where $\alpha_{g}^{*}$ is the fixed point value of the gauge coupling, taking $x_{F} \rightarrow 21 / 4$ ).

Note that we do not require all the couplings to be nonzero in order to have a nontrivial UV fixed point, but we definitely need to reproduce the gauge-Yukawa behavior of [16] that was observed in [2], while at the same time having negative beta functions for the couplings that are required to be nonzero in the IR, for phenomenological reasons. Therefore we can reject the Gaussian fixed point A. The second of these options, fixed point B, was the LS fixed point that was utilized in [2], and leads to

$$
\mathrm{B}: \frac{\beta_{Y}}{\alpha_{Y}}=\frac{\beta_{\tilde{Y}}}{\alpha_{\tilde{Y}}} \approx-\frac{3}{1+x_{F}} \alpha_{g} \rightarrow-\frac{12}{25} \alpha_{g}<0,
$$

so that both the $Y$ and $\tilde{Y}$ couplings flow away from fixed point $\mathrm{B}$ in the IR. Hence this fixed point is an interesting Gaussian option for the $Y$ and $\tilde{Y}$ couplings. In order to assess the other possible fixed points, note that

$$
\frac{\beta_{Y}-\beta_{\tilde{Y}}}{\alpha_{Y}-\alpha_{\tilde{Y}}}=2\left(1+x_{F}\right)\left(\alpha_{Y}+\alpha_{\tilde{Y}}\right)>0 .
$$

Hence $\alpha_{Y}-\alpha_{\tilde{Y}}$ shrinks in the IR, so if the flow begins in the $\mathrm{UV}$ at $\mathrm{C}$ or $\mathrm{D}$, it will be attracted to fixed point $\mathrm{E}$. We conclude that from the perspective of the couplings $g, y, \tilde{y}, Y, \tilde{Y}$, any of B,C,D,E are suitable for an asympotically safe fixed point but, as it flows to the IR, the system is attracted to the trajectory emerging from fixed point E, driven by the Yukawa couplings $Y, \tilde{Y}$. A numerical evolution showing this crossover for the Yukawa and gauge couplings is shown in Fig. 3.

Next we turn to the scalar couplings. Their beta functions are given by 

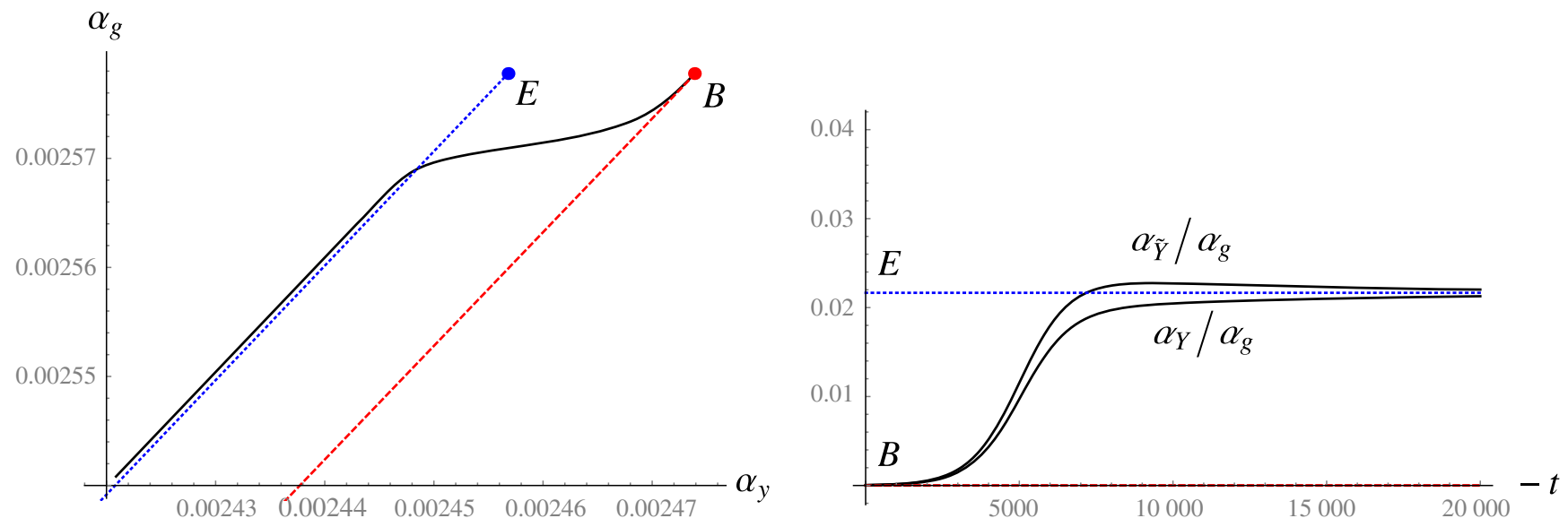

FIG. 3. The flow in gauge and Yukawa coupling-space from the true fixed point B on to the trajectory emanating from the pseudofixed E, as specified in Table III. During the flow the system crosses over from the (red-dashed) trajectory emanating from B, onto the bluedotted trajectory emanating from $\mathrm{E}$, inducing nonzero $\alpha_{Y}, \alpha_{\tilde{Y}}$. This evolution (which ignores the accompanying flow of the quartic couplings) is idealized: the system radiatively develops a minimum before reaching trajectory E.

$$
\begin{aligned}
\beta_{u_{1}}= & 4 \alpha_{u_{1}}\left[8 \alpha_{u_{1}}+8 \alpha_{u_{2}}+\left(\alpha_{y}+\alpha_{\tilde{y}}\right)\right]+32 \alpha_{v_{1}}^{2} x_{F}^{2}+6 \alpha_{u_{2}}^{2}, \\
\beta_{u_{2}}= & 2 \alpha_{u_{2}}\left[4 \alpha_{u_{2}}+\left(\alpha_{y}+\alpha_{\tilde{y}}\right)\right]-\frac{1}{2} x_{F}\left(\alpha_{y}^{2}+\alpha_{\tilde{y}}^{2}\right), \\
\beta_{w_{1}}= & 4 \alpha_{w_{1}}\left[8 \alpha_{w_{1}}+24 \alpha_{w_{2}}+2 x_{F}\left(\alpha_{Y}+\alpha_{\tilde{Y}}\right)-3 \alpha_{g}\right] \\
& +32 \alpha_{v_{1}}^{2} x_{F}^{2}+48 \alpha_{w_{2}}^{2}+\frac{3}{8} \alpha_{g}^{2}, \\
\beta_{w_{2}}= & 2 \alpha_{w_{2}}\left[12 \alpha_{w_{2}}+2 x_{F}\left(\alpha_{Y}+\alpha_{\tilde{Y}}\right)-3 \alpha_{g}\right] \\
& -\frac{1}{2} x_{F}\left(\alpha_{Y}^{2}+\alpha_{\tilde{Y}}^{2}\right)+\frac{3}{16} \alpha_{g}^{2}, \\
\beta_{v_{1}}= & 2 \alpha_{v_{1}}\left[16 \alpha_{u_{1}}+8 \alpha_{u_{2}}+16 \alpha_{w_{1}}+24 \alpha_{w_{2}}\right. \\
& \left.+\left(\alpha_{y}+\alpha_{\tilde{y}}\right)+2 x_{F}\left(\alpha_{Y}+\alpha_{\tilde{Y}}\right)-3 \alpha_{g}\right] \\
& -\frac{1}{2}\left(\alpha_{y}+\alpha_{\tilde{y}}\right)\left(\alpha_{Y}+\alpha_{\tilde{Y}}\right)-\Upsilon .
\end{aligned}
$$

Analysis of these renormalization group equations shows that there is only a real solution for a fixed point when $\alpha_{Y}=\alpha_{\tilde{Y}}=0$, corresponding to fixed point $\mathrm{B}$, namely the original LS fixed point studied in [2]. Along the trajectory from $\mathrm{B}$, the couplings assume the following values (with actually two stable branches for $w_{1}$ ):

$$
\begin{aligned}
& \alpha_{u_{1}}=\frac{-6 \sqrt{22}+3 \sqrt{19+6 \sqrt{22}}}{100} \alpha_{g}, \\
& \alpha_{u_{2}}=\frac{3}{25}(\sqrt{22}-1) \alpha_{g}, \\
& \alpha_{w_{1}}=\frac{3 \pm \sqrt{3(4 \sqrt{2}-5)}}{16 \sqrt{2}} \alpha_{g}, \\
& \alpha_{w_{2}}=\frac{1}{16}(2-\sqrt{2}) \alpha_{g}, \\
& \alpha_{v_{1}}=0 .
\end{aligned}
$$

It is important for later reference that, as discussed in $[16,17]$, the negative value of $\alpha_{u_{1}}$ at the minimum does not induce instability in $H$ because it is offset by the much larger positive value of $\alpha_{u_{2}}$.

Once the Yukawa flow in Fig. 3 begins, the scalar couplings also begin to flow: indeed Fig. 3 is somewhat idealized in the sense that the quartic couplings now rapidly induce radiative symmetry breaking. In order to show this analytically, one may use the relations between the Yukawas and gauge couplings corresponding to trajectory $E$ which yields an effective set of beta functions,

$$
\begin{aligned}
\beta_{u_{1}}= & 32 \alpha_{u_{1}}^{2}+6 \alpha_{u_{2}}^{2}+32 \alpha_{u_{1}} \alpha_{u_{2}}+\frac{1056}{277} \alpha_{u_{1}} \alpha_{g}, \\
\beta_{u_{2}}= & 8 \alpha_{u_{2}}^{2}+\frac{528}{277} \alpha_{u_{2}} \alpha_{g}-\frac{182952}{76729} \alpha_{g}^{2}, \\
\beta_{w_{1}}= & 32 \alpha_{w_{1}}^{2}+48 \alpha_{w_{2}}^{2}+96 \alpha_{w_{1}} \alpha_{w_{2}}-\frac{2820}{277} \alpha_{w_{1}} \alpha_{g}+\frac{3}{8} \alpha_{g}^{2}, \\
\beta_{w_{2}}= & 24 \alpha_{w_{2}}^{2}-\frac{1410}{277} \alpha_{w_{2}} \alpha_{g}+\frac{227163}{1227664} \alpha_{g}^{2}, \\
\beta_{v_{1}}= & 16 \alpha_{v_{1}}\left(2 \alpha_{u_{1}}+\alpha_{u_{2}}+2 \alpha_{w_{1}}+3 \alpha_{w_{2}}-\frac{441}{2216} \alpha_{g}\right) \\
& -\frac{1584}{76729} \alpha_{g}^{2} .
\end{aligned}
$$

These show that, once the system is kicked onto the $\mathrm{E}$ trajectory, the quartic couplings $u_{2}$ and $w_{2}$ flow to "quasifixed points", that is trajectories that are determined entirely by the slowly varying value of $\alpha_{g}$. Indeed $\alpha_{g}$ is parametrically slowly flowing compared to the quartics (because its beta function is order $\epsilon^{2}$ ), so we may approximate it as constant, with the quartic couplings starting close to the boundary values in (17). Solving for 

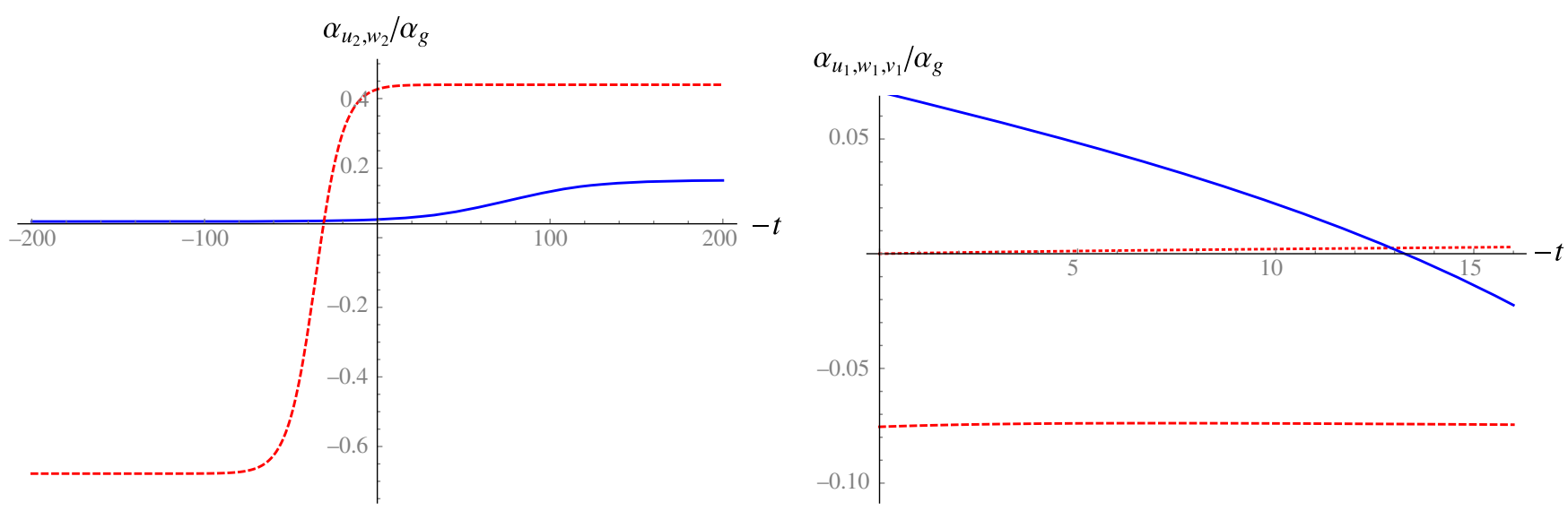

FIG. 4. The flow for the quartic couplings once the theory leaves trajectory B (at $t=0$ ). On the left, $\alpha_{u_{2}}$ in red/dashed and $\alpha_{w_{2}}$ in blue/ solid both flow to new fixed points (beginning from positive values at $t=0$ ). The $\alpha_{w_{2}}$ quasifixed point is much larger, which among other contributions induces $\alpha_{w_{1}}$ in blue/solid (on the right) to run negative and form a minimum radiatively for $\tilde{S}$. Meanwhile $\alpha_{u_{1}}$ in red/ dashed on the right is only moderately changed, not enough to destabilize $H$, while $\alpha_{v_{1}}$ in red/dotted runs slightly positive, inducing a small positive mass-squared for the Higgs at the PS breaking scale. This example has $\epsilon=0.01$.

$\alpha_{u_{2}}$ and $\alpha_{w_{2}}$ we see that they can both asymptote (as tanh functions) to positive "quasifixed" IR values given by ${ }^{2}$

$$
\begin{aligned}
\frac{\alpha_{u_{2}}}{\alpha_{g}} & \approx-\frac{33}{277}\left(1+\sqrt{22} \tanh \left(\frac{264 \sqrt{22}}{277} \frac{\Delta t}{\alpha_{g}}\right)\right) \\
& \rightarrow \frac{33}{277}(\sqrt{22}-1), \\
\frac{\alpha_{w_{2}}}{\alpha_{g}} & \approx \frac{1}{4432}\left(470-\sqrt{69458} \tanh \left(\frac{3 \sqrt{34729}}{277 \sqrt{2}} \frac{\Delta t}{\alpha_{g}}\right)\right) \\
& \rightarrow \frac{1}{4432}(\sqrt{69458}+470) .
\end{aligned}
$$

While the first quasifixed value for $u_{2}$ is very close to its starting point in (17), the quasifixed value for $w_{2}$ is much larger. (We should repeat that the analysis is approximate because the Yukawa couplings have not yet reached their new trajectory; in a full numerical evolution the running of the quartics will be delayed because the Yukawas have not yet reached trajectory $\mathrm{E}$, but it is expected to be qualitatively the same.) This evolution is shown numerically in the left panel of Fig. 4. In the right panel we show the effect on the remaining couplings $u_{1}, w_{1}, v_{1}$. Because $w_{2}$ appears only in the renormalization group equation for $w_{1}$, the two couplings $u_{1}$ and $v_{1}$ are changed only very slightly, with $v_{1}$ becoming slightly positive. Importantly no instability can be induced radiatively for $H$ at this stage, because (as was the case above on trajectory B) the negative contribution of $u_{1}$ to the potential is still offset by the positive contribution of $u_{2}$. On the other hand $w_{2}$ runs more positive in the IR,

\footnotetext{
${ }^{2} \mathrm{~A}$ more sophisticated treatment is possible, and other flows are possible, but this prescription is sufficient for a qualitative understanding.
}

and as can be seen from (18), this is a positive contribution to $\beta_{w_{1}}$, adding to several other positive contribution to $\beta_{w_{1}}$. The net result is that $w_{1}$ runs negative (regardless of $w_{2}$ in fact) and inevitably at some point overcomes the positive approximately constant contribution to the potential from the $w_{2}$ term itself, forming a radiative minimum as in Fig. 5 (as per [32]).

Thus (extended) PS breaking is induced radiatively in the $\mathrm{TM}$, and at this scale a small positive mass-squared is generated via the $v_{1}$ "portal" coupling. It is natural for the latter to then be driven negative itself below the PS breaking scale, due to the coupling of $H$ to the $N_{F}-n_{g} N_{S} \approx \frac{9}{4} N_{C}$ pairs of $Q, \tilde{Q}$ fields that remain light because (by chiral symmetry) they cannot receive a mass from the $Y, \tilde{Y}$ couplings. This flow would be similar to that of the other mass-squared operators above the scale of PS breaking which we discuss in the following section: a more complete analysis of the running of the "portal" Higgs

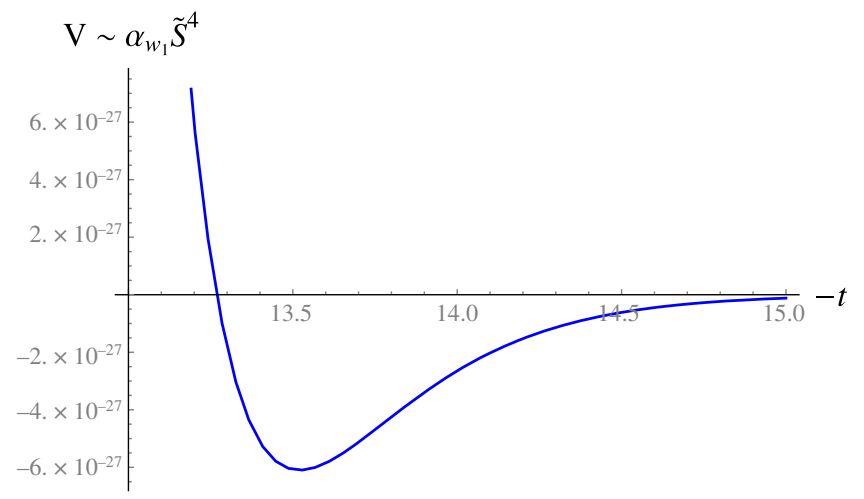

FIG. 5. The radiatively induced minimum in the effective potential for $\tilde{S}$. 
mass-squared below the PS scale will be undertaken in a later phenomenological study.

\section{B. The behavior of the relevant couplings-emergent flavor hierarchies from flavor symmetric fixed points}

We now turn to the behavior of classically relevant operators. These are allowed in the model as they are unable to disrupt the UV fixed point. (In any asymptotically safe theory such classically relevant operators are simply part of the collection of nonpredictive parameters in the theory.) They renormalize multiplicatively and can themselves initiate radiative symmetry breaking, as described in [1]. As in the minimal supersymmetric SM, one can begin with a set of entirely positive mass-squareds in the UV and have them run negative due to the large Yukawa couplings in the model.

In this section we shall perform a more complete analysis of the flow of these operators to show how one should incorporate their flavor dependence. In particular we are interested in the possible generation of flavor/generation hierarchies in $H$, which in any viable model will be required to satisfy phenomenological constraints.

Rather than write the explicit flavor dependence as in (3), we wish to consider smaller flavor structures that are closed under RG flow. To see how to do this, as a warm-up consider the completely $S U\left(N_{F}\right)$ symmetric terms in [2], which were mass-squareds of the form

$$
\begin{aligned}
M_{H_{i j} H_{k l}^{*}}^{2} & =m_{0}^{2} \delta_{j l} \delta_{i k}+2 \Delta^{2} \sum_{a} T_{j i}^{a} T_{k l}^{a} \\
& =m_{0}^{2} \delta_{j l} \delta_{i k}+\Delta^{2}\left(\delta_{j l} \delta_{i k}-\frac{1}{N_{F}} \delta_{j i} \delta_{k l}\right) .
\end{aligned}
$$

Defining real and imaginary parts, $H_{i j}=\frac{1}{\sqrt{2}}\left(h_{i j}+i p_{i j}\right)$, and $^{3} \quad h_{a}+i p_{a}=\sqrt{2} T_{i j}^{a}\left(h_{i j}+i p_{i j}\right)$, the corresponding operators can be written as

$$
\begin{aligned}
m_{0}^{2} H^{\dagger} H & =\frac{m_{0}^{2}}{2} \operatorname{Tr}\left(h^{2}+p^{2}\right), \\
\sum_{a} \frac{\Delta^{2}}{2}\left(h_{a}^{2}+p_{a}^{2}\right) & =\sum_{a} 2 \Delta^{2} \operatorname{Tr}\left(T_{a} H\right) \operatorname{Tr}\left(T_{a} H^{\dagger}\right) \\
& =\frac{\Delta^{2}}{2}\left[\operatorname{Tr}\left(h^{2}+p^{2}\right)-\frac{(\operatorname{Tr} h)^{2}+(\operatorname{Tr} p)^{2}}{N_{F}}\right] .
\end{aligned}
$$

It is now clear that one can proceed to break flavor in a way that commutes with the RG equations, by arranging the flavor breaking in $S U(n)$ subgroups, where the $S U(n)$

\footnotetext{
${ }^{3}$ Note that $h_{a}$ and $p_{a}$ are not simply related to $h_{i j}$ and $p_{i j}$. That is, while $p_{a}$ is the coefficient of the anti-Hermitian parts of $H, p_{i j}$ is the coefficient of the imaginary parts of $H$.
}

generators are in the $n \times n$ upper-left $n \times n$ block of the parent $S U\left(N_{F}\right)$, where $1<n \leq N_{F}$. This gives degenerate masses for the generators of each nested $S U(n)$ flavor subgroup, where we envisage an explicit breaking

$$
S U\left(N_{F}\right) \supset S U\left(N_{F}-1\right) \ldots \supset S U(n) \ldots
$$

So without loss of generality we can express the new Cartan generators introduced for each $S U(n)$ as

$$
T_{i j}^{\left(n^{2}-1\right)}=\frac{1}{\sqrt{2 n(n-1)}}\left(\begin{array}{cccccc}
1 & & & & & \\
& \ddots & & & & \\
& & 1 & & & \\
& & 1-n & \\
& & & 0 & \\
& & & & \ddots
\end{array}\right) \text {, }
$$

with the non-Cartan generators being defined accordingly in the obvious way. Defining the trace over the $S U(n)$ block of the generators as

$$
\operatorname{Tr}_{n}\left(\mathcal{O}_{i j}\right)=\sum_{i=1}^{n} \mathcal{O}_{i i}
$$

the flavor breaking generalization of (21) becomes

$$
\begin{aligned}
V^{(2)}= & \frac{m_{0}^{2}}{2} \operatorname{Tr}_{N_{F}}\left(h^{2}+p^{2}\right)+\sum_{n=1}^{N_{F}-1} \frac{m_{n}^{2}}{2}\left[\frac{\left(\operatorname{Tr}_{n} h\right)^{2}+\left(\operatorname{Tr}_{n} p\right)^{2}}{n}\right] \\
& +\sum_{n=2}^{N_{F}} \frac{\Delta_{n}^{2}}{2}\left[\operatorname{Tr}_{n}\left(h^{2}+p^{2}\right)-\frac{\left(\operatorname{Tr}_{n} h\right)^{2}+\left(\operatorname{Tr}_{n} p\right)^{2}}{n}\right] .
\end{aligned}
$$

These operators form a system closed under RG flow, and we may now determine their coefficients in $16 \pi^{2} \partial_{t} V$, relevant for solving the Callan-Symanzik equation: these are shown in Table IV. One can now solve the RG equations along trajectory B for these parameters to see how they evolve before their flow is cut off by the radiative symmetry breaking (regardless of how it arises): as for any relevant parameter the flow will be expressed in terms of a set of RG-invariants. In this case, defining $f_{y}=\alpha_{y} / \alpha_{g} \approx 0.46$, $f_{u_{1}}=\alpha_{u_{1}} / \alpha_{g} \approx-0.30, f_{u_{2}}=\alpha_{u_{2}} / \alpha_{g} \approx 0.44$, and 
TABLE IV. The relevant quadratic operators and their beta function coefficients in terms of the quartic $u_{1,2}$ couplings.

\begin{tabular}{lcl}
\hline \hline Coupling & \multicolumn{1}{c}{ Operator } & \multicolumn{1}{c}{ Coefficient in $16 \pi^{2} \partial_{t} V$} \\
\hline$m_{0}^{2}$ & $\operatorname{Tr}_{N_{F}}\left(h^{2}+p^{2}\right)$ & $m_{0}^{2}\left\{2 u_{1}\left[N_{F}^{2}+1\right]+4 u_{2} N_{F}\right\}+\Delta_{N_{F}}^{2}\left(2 u_{1}+\frac{4 u_{2}}{N_{F}}\right)\left(N_{F}^{2}-1\right)$ \\
& & $+\sum_{n}^{N_{F}-1} 2 u_{1}\left(m_{n}^{2}+\Delta_{n}^{2}\left(n^{2}-1\right)\right)$ \\
$\Delta_{N_{F}}^{2}$ & $\operatorname{Tr}_{N_{F}}\left(h^{2}+p^{2}\right)-\frac{\left(\operatorname{Tr}_{N_{F}} h\right)^{2}+\left(\operatorname{Tr}_{N_{F}} p\right)^{2}}{N_{F}}$ & $2 u_{1} \Delta_{N_{F}}^{2}$ \\
$\Delta_{n}^{2}$ & $\operatorname{Tr}_{n}\left(h^{2}+p^{2}\right)-\frac{\left(\operatorname{Tr}_{n} h\right)^{2}+\left(\operatorname{Tr}_{n} p\right)^{2}}{n}$ & $2 u_{1} \Delta_{n}^{2}+\frac{4 u_{2}}{n}\left(m_{n}^{2}+\Delta_{n}^{2}\left(n^{2}-1\right)\right)$ \\
$m_{n}^{2}$ & $\frac{\left(\operatorname{Tr}_{n} h\right)^{2}+\left(\operatorname{Tr}_{n} p\right)^{2}}{n}$ & $2 u_{1} m_{n}^{2}+\frac{4 u_{2}}{n}\left(m_{n}^{2}+\Delta_{n}^{2}\left(n^{2}-1\right)\right)$ \\
\hline \hline
\end{tabular}

$$
\begin{aligned}
f & =2 f_{y}+4 f_{u_{1}}\left(1+\frac{1}{N_{F}^{2}}\right)+8 f_{u_{2}} \approx 3.22, \\
f_{\Delta} & =2 f_{y}+\frac{4}{N_{F}^{2}} f_{u_{1}} \approx 0.92, \\
f_{n} & =8 f_{u_{2}} \frac{n}{N_{F}}\left(1-\delta_{n N_{F}}\right),
\end{aligned}
$$

the RG-invariants are found to be

$$
\begin{gathered}
\tilde{m}_{*}^{2}=\tilde{m}^{2}(0)(\tilde{\Omega}(0))^{-f}, \\
\sigma_{n *}^{2}=\left[m_{n}^{2}(0)+\left(n^{2}-1\right) \Delta_{n}^{2}(0)\right](\tilde{\Omega}(0))^{-\left(f_{\Delta}+f_{n}\right)}, \\
\rho_{n *}^{2}=\left[\Delta_{n}^{2}(0)-m_{n}^{2}(0)\right](\tilde{\Omega}(0))^{-f_{\Delta}},
\end{gathered}
$$

where

$$
\tilde{\Omega}(t)=\left(\frac{\alpha_{g}^{*}}{\alpha_{g}}-1\right)^{-3 / 4 \epsilon} .
$$

In terms of these we find the following solutions for the operators in (24):

$m_{0}^{2}=\left(\frac{\tilde{\Omega}(t)}{\tilde{\Omega}(0)}\right)^{f} \tilde{m}_{*}^{2}-\frac{1}{N_{F}^{2}} \sum_{n}^{N_{F}} \frac{\sigma_{n *}^{2}}{1+2 \frac{f_{u_{2}}}{f_{u_{1}}}\left(1-n / N_{F}\right)} \tilde{\Omega}^{f_{\Delta}+f_{n}}$

$\Delta_{n}^{2}=\frac{1}{n^{2}}\left(\rho_{n *}^{2} \tilde{\Omega}^{f_{\Delta}}+\sigma_{n *}^{2} \tilde{\Omega}^{f_{\Delta}+f_{n}}\right)$,

$m_{n}^{2}=\frac{1}{n^{2}}\left(\rho_{n *}^{2}\left(1-n^{2}\right) \tilde{\Omega}^{f_{\Delta}}+\sigma_{n *}^{2} \tilde{\Omega}^{f_{\Delta}+f_{n}}\right)$.

This is the desired form, since it assumes nothing about the "starting values", which are simply values chosen at an arbitrary point in renormalization time, and it properly encapsulates all the nonpredictive parameters in the theory. The entire flow is determined by these parameters and $\tilde{\Omega}(t)$, which just determines where one is in renormalization time.

The interesting feature of these solutions is that $\tilde{\Omega} \rightarrow 0$ in the IR. Simple flavor hierarchies can therefore be generated much like the mechanism for radiative symmetry breaking in [1]. That is the exponent $f$ is much larger than $f_{n}$ or $f_{\Delta}$.
Therefore $m_{0}^{2}$ runs to zero in the IR much more quickly than $\Delta_{n}^{2}$ or $m_{n}^{2}$. Meanwhile in the deep IR one can see from these solutions and the corresponding operators in Table IV, that hierarchies are naturally driven into the trace components in the potential which becomes dominant,

$V \rightarrow \sum_{n>1} \Delta_{n}^{2}\left[\operatorname{Tr}_{n}\left(h^{2}+p^{2}\right)-n\left(\left(\operatorname{Tr}_{n} h\right)^{2}+\left(\operatorname{Tr}_{n} p\right)^{2}\right)\right]$

This supports the intriguing possibility that flavor hierarchies originate within the VEVs of the Higgs sector, which would themselves become correspondingly hierarchical.

\section{CONCLUSIONS}

In this paper we have presented a model, the Tetrad Model (TM), which is asymptotically safe, and which descends directly to the Standard Model via radiative symmetry breaking. In terms of a convenient "quiverlike" interpretation, the model contains 4-units, with matter and electroweak Higgs fields falling into an extended PatiSalam GUT structure, based on the gauge group $S U\left(N_{C}\right) \times S U(2)_{L} \times S U(2)_{R}$, and a fourth unit that provides the PS breaking. (The electroweak gauging of a subgroup of the flavor symmetry can not be shown on the quiver, but nevertheless the language is useful for understanding the overall structure of the gauge-Yukawa UV fixed point.) At low energies the model is able to yield the Standard Model enhanced only in the Higgs fields, which carry the same generation indices as the matter fields.

Remarkably radiative symmetry breaking (i.e., the Coleman-Weinberg mechanism) operates in the model with no further adjustment. The (extended) PS Higgs naturally develops a VEV radiatively while the electroweak Higgs gains a positive "boundary value" mass-squared at the PS scale, due to a portal coupling that runs from zero at the UV fixed point. This mass-squared can itself then be driven negative in the IR. It was also found that it is natural to generate hierarchies among the electroweak Higgs VEVs due to the enhancement of mass-squared hierarchies as the theory runs to the IR.

How close to the Standard Model does the final result come? And what remains to be done? The appearance of 
the Coleman-Weinberg mechanism for the breaking of the enhanced PS GUT symmetry is certainly a welcome first step on the road towards the pure SM. It removes all signs of gauge symmetry enhancement above the PS scale. In order to induce reasonable mass-squared operators for the electroweak sector, this scale is most naturally around $100 \mathrm{TeV}$. What then remains below the PS scale is an electroweak sector augmented by $N_{F}$ vectorlike pairs of Higgsinolike (the $q, \tilde{q}$ states) doublets (with charges $0, \pm 1$ ). These states get masses from the electroweak Higgses so by measuring them we can learn about the enhanced Higgs sector. The main phenomenological question then lies in this latter point, namely which electroweak Higgses get VEVs and masses. As we saw the Higgses carry the same generation indices that matter does. Therefore, as usual for multi-Higgs models, while we have an attractive mechanism for generating hierarchies, one must be careful to avoid flavor constraints. The nonflavored block (i.e., $H_{0}$ ) can be safely given VEVs and mass-squareds, removing both $H_{0}$ itself from the spectrum and most of the vectorlike states. This leaves a $3 \times 3$ set of light Higgs doublets that must be handled more carefully. Since we may give these fields mass-squareds arbitrarily (they are after all relevant operators and hence free parameters of the model), the real question is then how to generate the VEVs dynamically without violating constraints, in particular tree-level flavor changing neutral currents. Studies with multi-Higgs models are summarized in Ref. [36]: generally the picture is acceptable if there is a dominant source of electroweak breaking, that dominantly couples to the matter fields. The simplest way to achieve this seems to be to use the masssquared parameters to lift the masses of all but one Higgs which dominantly couples to the third generation. Then lighter masses are generated through mixings. Intriguingly the structure of the low-energy theory can be similar to the minimal model of fermion masses found in [37], with the colored $q, \tilde{q}$ states providing the required massive vectorlike quarks, with masses of order the PS-scale. The flavor hierarchies would then naturally be related to the weak/PS hierarchy. It would be interesting to carry out a comprehensive study of this sector.

Finally we should remark that throughout we have been presenting the analysis in terms of the Veneziano limit in which the number of colors and flavors is arbitrarily large and the coupling at the UV fixed point arbitrarily weak. In practice one expects there to be a phase diagram for the UV behavior with more strongly coupled asymptotically safe behavior being exhibited with reasonable numbers of colors and flavors inside a conformal window, along the lines laid out in Ref. [38].

\section{ACKNOWLEDGMENTS}

It is a pleasure to thank Giacomo Cacciapaglia, Daniel Litim, Zhi Wei Wang, and Christoph Wetterich for comments and discussions. This work is partially supported by the Danish National Research Foundation under Grant No. DNRF:90.
[1] S. Abel and F. Sannino, Radiative symmetry breaking from interacting UV fixed points, Phys. Rev. D 96, 056028 (2017).

[2] S. Abel and F. Sannino, Framework for an asymptotically safe Standard Model via dynamical breaking, Phys. Rev. D 96, 055021 (2017).

[3] S. Weinberg, in C76-07-23.1 HUTP-76/160, Erice Sub-nucl. Phys., 1 (1976).

[4] S. P. Martin and J. D. Wells, Constraints on ultraviolet stable fixed points in supersymmetric gauge theories, Phys. Rev. D 64, 036010 (2001).

[5] H. Gies, J. Jaeckel, and C. Wetterich, Towards a renormalisable standard model without fundamental Higgs scalar, Phys. Rev. D 69, 105008 (2004).

[6] M. Shaposhnikov and D. Zenhausern, Quantum scale invariance, cosmological constant and hierarchy problem, Phys. Lett. B 671, 162 (2009).

[7] H. Gies, S. Rechenberger, and M. M. Scherer, Towards an asymptotic-safety scenario for chiral Yukawa systems, Eur. Phys. J. C 66, 403 (2010).

[8] J. Braun, H. Gies, and D. D. Scherer, Asymptotic safety: A simple example, Phys. Rev. D 83, 085012 (2011).
[9] F. Bazzocchi, M. Fabbrichesi, R. Percacci, A. Tonero, and L. Vecchi, Fermions and Goldstone bosons in an asymptotically safe model, Phys. Lett. B 705, 388 (2011).

[10] C. Wetterich, Where to look for solving the gauge hierarchy problem?, Phys. Lett. B 718, 573 (2012).

[11] Z. Chacko and R. K. Mishra, Effective theory of a light Dilaton, Phys. Rev. D 87, 115006 (2013).

[12] O. Antipin, M. Gillioz, E. Mølgaard, and F. Sannino, The a theorem for Gauge-Yukawa theories beyond Banks-Zaks, Phys. Rev. D 87, 125017 (2013).

[13] H. Gies, S. Rechenberger, M. M. Scherer, and L. Zambelli, An asymptotic safety scenario for gauged chiral HiggsYukawa models, Eur. Phys. J. C 73, 2652 (2013).

[14] G. Marques Tavares, M. Schmaltz, and W. Skiba, Higgs mass naturalness and scale invariance in the UV, Phys. Rev. D 89, 015009 (2014).

[15] S. Abel and A. Mariotti, Novel Higgs potentials from gauge mediation of exact scale breaking, Phys. Rev. D 89, 125018 (2014).

[16] D. F. Litim and F. Sannino, Asymptotic safety guaranteed, J. High Energy Phys. 12 (2014) 178. 
[17] D. F. Litim, M. Mojaza, and F. Sannino, Vacuum stability of asymptotically safe gauge-Yukawa theories, J. High Energy Phys. 01 (2016) 081.

[18] K. Intriligator and F. Sannino, Supersymmetric asymptotic safety is not guaranteed, J. High Energy Phys. 11 (2015) 023.

[19] A. D. Bond and D. F. Litim, Theorems for asymptotic safety of gauge theories, Eur. Phys. J. C 77, 429 (2017); Erratum 77, 525(E) (2017).

[20] G. M. Pelaggi, A. D. Plascencia, A. Salvio, F. Sannino, J. Smirnov, and A. Strumia, Asymptotically safe standard model extensions?, Phys. Rev. D 97, 095013 (2018).

[21] A. D. Bond, G. Hiller, K. Kowalska, and D. F. Litim, Directions for model building from asymptotic safety, J. High Energy Phys. 08 (2017) 004.

[22] D. Barducci, M. Fabbrichesi, C. M. Nieto, R. Percacci, and V. Skrinjar, In search of a UV completion of the standard model-378,000 models that don't work, J. High Energy Phys. 11 (2018) 057.

[23] D. F. Litim, Renormalisation group and the Planck scale, Phil. Trans. R. Soc. A 369, 2759 (2011).

[24] A. Eichhorn, An asymptotically safe guide to quantum gravity and matter, arXiv:1810.07615.

[25] J. Gracey, The QCD $\beta$-function at $\mathrm{O}\left(1 \mathrm{~N}_{\mathrm{F}}\right)$, Phys. Lett. B 373, 178 (1996); A. Palanques Mestre and P. Pascual, The $1 / N_{f}$ expansion of the $\gamma$ and $\beta$ functions in Q.E.D., Commun. Math. Phys. 95, 277 (1984); For a large nf review see, B. Holdom, Large $\mathrm{N}$ flavor $\beta$-functions: A recap, Phys. Lett. B 694, 74 (2010).

[26] C. Pica and F. Sannino, UV and IR zeros of gauge theories at the four loop order and beyond, Phys. Rev. D 83, 035013 (2011).
[27] E. Molinaro, F. Sannino, and Z. W. Wang, Safe Pati-Salam, Phys. Rev. D 98, 115007 (2018).

[28] O. Antipin, N. A. Dondi, F. Sannino, A. E. Thomsen, and Z. W. Wang, Gauge-Yukawa theories: Beta functions at large $N_{f}$, Phys. Rev. D 98, 016003 (2018).

[29] O. Antipin and F. Sannino, Conformal window 2.0: The large $N_{f}$ safe story, Phys. Rev. D 97, 116007 (2018).

[30] R. Mann, J. Meffe, F. Sannino, T. Steele, Z. W. Wang, and C. Zhang, Asymptotically Safe Standard Model via Vectorlike Fermions, Phys. Rev. Lett. 119, 261802 (2017).

[31] S. R. Coleman and E. J. Weinberg, Radiative corrections as the origin of spontaneous symmetry breaking, Phys. Rev. D 7, 1888 (1973).

[32] E. Gildener, Radiatively induced spontaneous symmetry breaking for asymptotically free gauge theories, Phys. Rev. D 13, 1025 (1976).

[33] E. Gildener and S. Weinberg, Symmetry breaking and scalar bosons, Phys. Rev. D 13, 3333 (1976).

[34] L. E. Ibanez and G. G. Ross, $S U(2)_{L} \times U(1)$ symmetry breaking as a radiative effect of supersymmetry breaking in guts, Phys. Lett. 110B, 215 (1982).

[35] S. F. King and Q. Shafi, Minimal supersymmetric $S U(4) \times S U(2)_{L} \times S U(2)_{R}$, Phys. Lett. B 422, 135 (1998).

[36] R. S. Gupta and J. D. Wells, Next generation Higgs bosons: Theory, constraints and discovery prospects at the large hadron collider, Phys. Rev. D 81, 055012 (2010).

[37] A. J. Buras, C. Grojean, S. Pokorski, and R. Ziegler, FCNC effects in a minimal theory of fermion masses, J. High Energy Phys. 08 (2011) 028.

[38] A. D. Bond, D. F. Litim, G. Medina Vazquez, and T. Steudtner, UV conformal window for asymptotic safety, Phys. Rev. D 97, 036019 (2018). 